\title{
When patient-centred care is worth doing well: informed consent or shared decision-making
}

\author{
Marleen Kunneman, ${ }^{1,2}$ Victor M Montori ${ }^{2}$
}

'Department of Medical Psychology, Academic Medical Center, University of Amsterdam, Amsterdam, The Netherlands ${ }^{2}$ Knowledge and Evaluation Research Unit, Mayo Clinic, Rochester, Minnesota, USA

\section{Correspondence to} Dr Victor M Montori, Knowledge and Evaluation Research Unit in Endocrinology, Mayo Clinic, 200 1st Street SW, Rochester MN 55905, USA;

montorivictor@mayo.edu

Accepted 7 September 2016 Published Online First

26 September 2016

\section{SLinked}

http://dx.doi.org/10.1136/ bmjqs-2016-005663

CrossMark

To cite: Kunneman $\mathrm{M}$, Montori VM. BMJ Qual Saf 2017;26:522-524.
High quality care is patient-centred. ${ }^{1}$ Efforts to promote patient-centred care in clinical practice should improve quality. Both shared decision-making (SDM) and the process of obtaining informed consent could be expressions of patient-centred care- to the extent that they respond to the advocates' call for 'nothing about me without me'. In this issue of BMJ Quality and Safety, Shahu et al discuss variations in the quality of informed consent procedures, which could, in their view, fail to support patient-centred care in general, and SDM specifically.

Readers interested in advancing this domain of quality may, therefore, be interested in improving the quality of informed consent procedures and promoting the implementation and routine use of SDM. But are these similar practices? Is informed consent a lesser version of SDM, with SDM the ideal expression of patient autonomy and involvement? Or are these different in purpose, process and outcomes?

Informed consent establishes a minimal legal standard in which (1) clinicians disclose the risks, benefits and alternatives of a proposed treatment or procedure and (2) people accept or reject this procedure that has been identified to be the most relevant for them. ${ }^{3}$ The patient involvement is limited to accepting or rejecting a proposed path. This 'choice' is implemented late in the trajectory of the patient and in the process of making decisions about care. By the time the process becomes recognisable to the parties and formal, often at the time of reviewing and signing an informed consent document, the decision to go forward has been made and is being implemented, perhaps even without active patient involvement.

Why then offer choice in this way to patients? The motivation of informed consent is legal, and its formal procedures reflect legal standards established in local jurisprudence. These standards include the detail with which other options should be presented, the format used and the process used to achieve consent, a process that should conclude with its documentation. In reality, as Shahu and colleagues note, this process, which may sound much like SDM, gets transformed into the collection of a signature on a document in which most often only one option is offered or described in any detail. ${ }^{2}$ Meeting the standard becomes the goal.

In contrary, SDM represents a more evolved response to the need for patient centeredness to the extent that it explicitly presents and stimulates to consider more than one option and helps patients and clinicians to deliberate on what is best. In a conversational dance, clinicians and patients work together to think, talk and feel through the situation of the patient and identify sensible ways to address this situation. ${ }^{4} 5$ The two-way information sharing in SDM, ${ }^{6} 7$ both by the clinician presenting the relevant options and the patient articulating what matters to him, is relevant for this particular patient and is relevant for comparing different options and choosing one that fits. In contrast, informed consent procedures seem independent of who this patient is and cover topics that are relevant for all people like this patient. ${ }^{5}$ Unlike the conversational dance in SDM that requires a human connection of careful and kind care, informed consent procedures call for a more technical and mechanical approach, a formal one-way provision of information in which boxes of topics addressed are to be actually or metaphorically ticked for administrative and legal purposes. While SDM seeks an answer to achieve resolution in the 
patient situation from several options available, informed consent procedures seek to obtain the 'go ahead' to implement one option.

This absence of recognition of more than one sensible way to address the patient's situation, so-called choice awareness, ${ }^{8} 9$ is a key limitation in the patient centeredness of informed consent procedures. Usually, the existence of other relevant and sensible actions, including foregoing active procedures, is not covered or including only cursorily within informed consent procedures. Shahu $e t a l^{2}$ report that none of the informed consent forms for cardiovascular procedures they analysed offered or described an alternative option.

This problem appears not to arise from the attention to detail that developers place in the construction of informed consent forms. We put forth, rather, that it is a manifestation of the culture of healthcare. In the context of adjuvant radiotherapy treatment, an observational study described the behaviour of radiation oncologists in terms that would suggest that they were applying informed consent principles, without the legal documents. Patients with early-stage rectal cancer could be treated with a number of approaches, including adjuvant radiation. Surgical oncologists would refer patients to discuss this possibility and its relative pros and cons with an expert radiation oncologist. These radiation oncologists reportedly favour an SDM approach and as a group they have endorsed in treatment guidelines the need for a shared decision. ${ }^{10} 11$ Yet, it was not made clear to patients that they were exploring one of several options as radiation oncologists almost always failed to create choice awareness and present more than one sensible option. In other words, they were obtaining patients' informed consent without a signature of legal value. ${ }^{8}$ Most often, radiation oncologists expressed the reason for the clinician-patient encounter to be for them to explain the one treatment they had to offer. Similar findings have been shown in other so-called 'preference sensitive' treatment decisions as well. ${ }^{8} 12$

A reason for using an informed consent process rather than inviting active patient involvement and using an SDM approach in which sensible options are discussed at length could be found in the referral process. ${ }^{9} 12$ When referring and treating clinicians, and patients alike, all believe that patients are referred because they need a certain treatment or procedure, none of them will initiate the necessary conversational dance inherent in SDM. Instead, they will go through the minimal requirements of informed consent as an attempt - a minimal one-to make care more patientcentred. Patients on their way to cardiac catheterisation, invited to sign a form, may find this to be transactional, routine, but certainly not an expression of centring care around them.

The use of transactional and technical approaches to making decisions about care, timid efforts that seek in situations in which SDM would be ideal fails the patient-centred goals of quality. It suggests that the focus is not on caring but on doing business, in making formal agreements and contracts. When SDM is the right response to address the patient's situation but instead the patient is provided with little more than a form this is a quality gap, regardless of the quality of the informed consent document.

Beyond legal minima, what should the quality movement seek? SDM should be a manifestation of advanced patient-centred care. In SDM, clinicians invite patients into a conversation in which they consider the options available that may help address the situation of the patient. In doing so, the SDM process presents the patient situation in high definition. It is pertinent when the right answer is not apparent before the conversation, when care depends on who the patient is and what is their situation. Options, with evidence-based features described in accessible ways (which may need to be made readily available to the parties, perhaps within the evolving electronic workflows of the practice), are carefully discussed until the best solution for this patient's situation becomes evident.

We must not confuse SDM with other forms of patient and family involvement in which we seek their consent to proceed with a course of action. We must not confuse SDM with the distribution of decision aids or patient education materials for patients, expecting patients to find the best answer on their own: there is nothing shared about choosing alone from a menu! In SDM, consent with a pre-established or expected course is not the goal, as it should also not be the end goal of our efforts to care. Our goal should be to seek the resolution of the patients' situation in a way that fits with their values, preferences and context. Like other things worth doing, SDM is worth doing well. And when it is done well, it should be recognised as high quality care.

Competing interests None declared.

Provenance and peer review Commissioned; internally peer reviewed.

\section{REFERENCES}

1 Institute of Medicine (US) Committee on Quality of Health Care in America. Crossing the quality chasm: a new health system for the 21st century. Washington, DC, 2001.

2 Shahu A, Schwartz J, Perez M, et al. Discerning quality: an analysis of informed consent documents for common cardiovascular procedures. BMJ Qual Saf 2017;26: 569-71.

3 Lindor RA, Kunneman M, Hanzel M, et al. Liability and informed consent in the context of shared decision making. Acad Emerg Med 2016;23:1428-33.

4 Hargraves I, LeBlanc A, Shah ND, et al. Shared decision making: the need for patient-clinician conversation, not just 


\section{Editorial}

5 Kunneman M, Montori VM, Castaneda-Guarderas A, et al. What is shared decision making? (and what it is not). Acad Emerg Med 2016;23:1320-4.

6 Charles C, Gafni A, Whelan T. Shared decision-making in the medical encounter: what does it mean? (or it takes at least two to tango). Soc Sci Med 1997;44:681-92.

7 Elwyn G, Frosch D, Thomson R, et al. Shared decision making: a model for clinical practice. J Gen Intern Med 2012;27:1361-7.

8 Kunneman M, Engelhardt EG, ten Hove FL, et al. Deciding about (neo-)adjuvant rectal and breast cancer treatment: missed opportunities for shared decision making. Acta Oncol 2016;55:134-9.
9 Kunneman M. Shared decision making in adjuvant cancer treatment. Leiden University Medical Center, 2016.

10 Pieterse AH, Stiggelbout AM, Baas-Thijssen MC, et al. Benefit from preoperative radiotherapy in rectal cancer treatment: disease-free patients' and oncologists' preferences. $\mathrm{Br} J$ Cancer 2007;97:717-24.

11 Netherlands CCCt. Guidelines for the management of colorectal cancer and colorectal liver metastases, 2014.

12 Snijders HS, Kunneman M, Bonsing BA, et al. Preoperative risk information and patient involvement in surgical treatment for rectal and sigmoid cancer. Colorectal Dis 2014;16:O43-9. 